\title{
A Semi-Blind Channel Estimation Method for Multiuser Multiantenna OFDM Systems
}

\author{
Yonghong Zeng, Member, IEEE, and Tung-Sang Ng, Fellow, IEEE
}

\begin{abstract}
A subspace-based blind method is proposed for estimating the channel responses of a multiuser and multiantenna orthogonal frequency division multiplexing (OFDM) uplink system. It gives estimations to all channel responses subject to a scalar matrix ambiguity and does not need precise channel order information (only an upper bound for the orders is required). Furthermore, the scalar ambiguity matrix can be easily resolved by using only one pilot OFDM block, given that the number of users is smaller than the number of symbols in the pilot symbol block. Equalization methods are discussed based on the estimated channels. By using partial knowledge of the channels, a multipath subspace method is proposed that reduces the computational complexity. Simulations show that the methods are effective and robust.
\end{abstract}

Index Terms-Blind channel identification, MIMO, multiantenna, multiuser, OFDM, semi-blind, subspace method, zero-padding.

\section{INTRODUCTION}

$\mathbf{M}$ ULTIMEDIA applications in wireless communication call for very high data rate transmissions. To meet this demand, various methods have been proposed [1]-[12]. Among them, orthogonal frequency division multiplexing (OFDM) and multiple-input multiple-output (MIMO) have emerged as two major techniques in the future fourth-generation (4G) communications [3], [10]. OFDM is a special case of multicarrier modulation, which can effectively mitigate the effects of multipath propagation and, hence, increase data rate [3]-[5], [13]-[15]. In addition, an OFDM system can be equalized in the frequency domain by using the fast Fourier transform (FFT), which is fast and efficient. Classically, the cyclic prefix (CP) is used in OFDM for combating multipath and achieving frequency domain equalization. We call it CP-OFDM for convenience. Recently, zero-padding OFDM (ZP-OFDM), which zero-pads rather than cyclicly prefixing each block, has been proposed [4], [14], [15]. ZP-OFDM not only has all the advantages of CP-OFDM but also avoids interblock interference (IBI). Without IBI, the channel estimation and equalization are simplified [4], [14], [15]. Another technique [single carrier with cyclic prefix (SCCP)] [16], [17] has nearly the same advantages of OFDM, but it avoids OFDM's shortcomings

Manuscript received August 26, 2002; revised June 16, 2003. Part of this work was presented at the IEEE International Symposium on Circuits and System (ISCAS), Bangkok, Thailand, May 2003. The associate editor coordinating the review of this manuscript and approving it for publication was Dr. Ta-Hsin $\mathrm{Li}$.

Y. Zeng is with the Department of Electrical and Electronic Engineering, The University of Hong Kong, Hong Kong, on leave from the National University of Defense Technology, Changsha, China (e-mail: yhzeng@eee.hku.hk).

T.-S. Ng is with the Department of Electrical and Electronic Engineering, The University of Hong Kong, Hong Kong (e-mail: tsng @eee.hku.hk).

Digital Object Identifier 10.1109/TSP.2004.826183 of high peak-to-average power ratio and high sensitivity to frequency errors [18]. It is easy to turn the SCCP to single carrier with zero-padding (SCZP), which uses zero-padding other than the cyclic prefix for each block as CP-OFDM turns to ZP-OFDM. SCZP shares all the advantages of SCCP and, furthermore, avoids IBI. Using multiple transmit and receive antennas has the potential to increase the channel capacity and, thus, maximize achievable data rate [1], [8], [10]. Combining MIMO and OFDM is, therefore, believed to have the ability to achieve better performance [10]. In recent years, systems using OFDM and/or multiple transmit and receive antennas have been studied extensively [2], [6], [7], [9], [11], [12], [15]. However, most of these studies concentrate on a single user with one or more transmitting and receiving antennas, sometimes with space-time coding. In [11], a multiuser and multiantenna OFDM system (MUMA-OFDM) is proposed, in which many users can share the same frequency band if multiple antennas are installed at the basestation. Each user's signal is modulated via OFDM. A joint detection method is presented based on the assumption that channel conditions are completely known. Unfortunately, no method to estimate the channel responses is given, which is known to be one of the most difficult tasks in a MIMO system.

Blind channel identification for MIMO linear system has been studied extensively in recent years [4], [19]-[32]. Among various known algorithms, second-order statistics (SOS)-based algorithms are most attractive due to their special properties [4], [21], [22]. Since the first SOS-based method for simple-input multiple-output (SIMO) systems, introduced by Tong et al. in [30], there have been a variety of SOS-based algorithms [4], [19], [20], [22]-[25], [27]-[32]. The subspace (SS) method [19], [28], [33] is believed to be one of the best. The SS method has a simple structure and achieves good performance in the SIMO system [28], but it requires precise knowledge of the channel order [4], [22], [33], which is very difficult to obtain in practice. In addition, the extension of it to the general MIMO system is not successful because it generally can only estimate the channels subject to a polynomial matrix ambiguity [19]. Unlike the SIMO case, it is much more difficult to estimate channel orders in the MIMO system, because not just one but many orders need to be estimated. Due to noise and roundoff errors, it is not possible to obtain precise channel orders, but usually, their upper bounds can be obtained. Therefore, the SS method is not practical for general MIMO channel estimation. Recently, some SS methods have been proposed for single-user OFDM systems [13], [34]. The method in [34] can be applied to OFDM systems without $\mathrm{CP}$ and, therefore, leads to higher data rate. However, similar to the SS method for general SIMO 


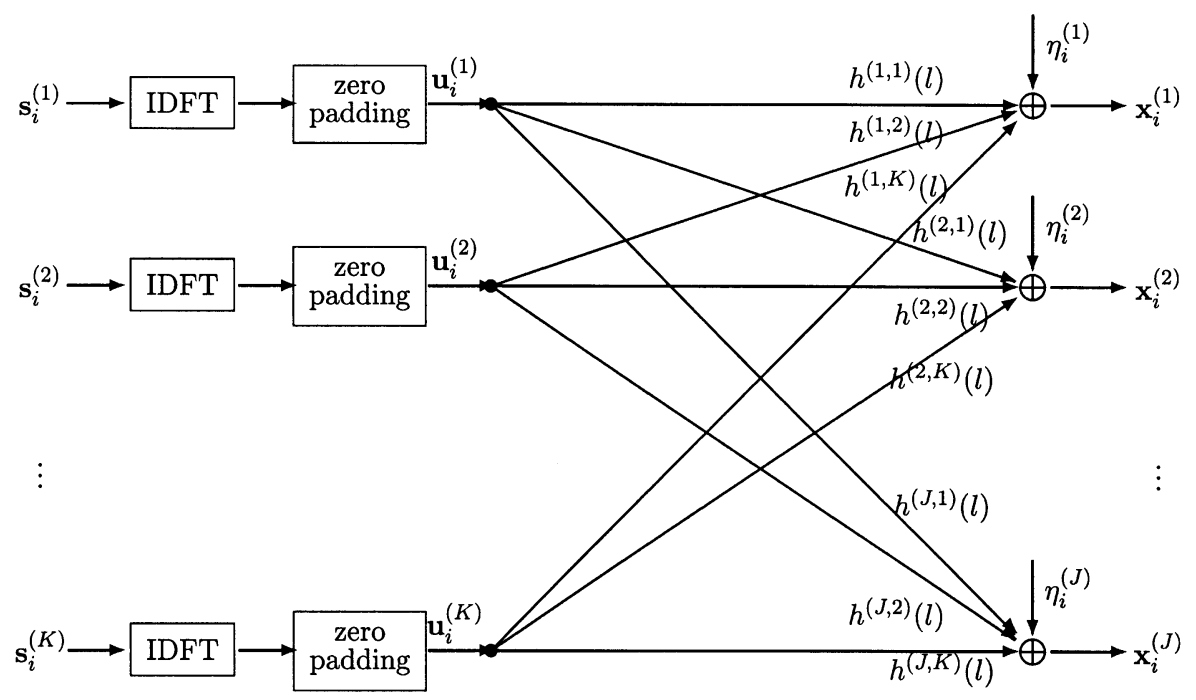

Fig. 1. Multiuser multiantenna OFDM system (uplink).

systems, it requires an exact channel order as a priori. The method in [13] successfully avoids the requirement of exact channel order by properly using the $\mathrm{CP}$ in CP-OFDM systems. The two methods can only be used for single-user OFDM systems, and their extension to multiuser systems is by no means straightforward.

In this paper, we propose a subspace method for estimating the MIMO channels in the uplink MUMA-OFDM system, where the ZP-OFDM [4], [15], and not the CP-OFDM, is used. The method is also valid for the MUMA-SCZP system. We will show that by making use the property of zero-padding, the proposed subspace method no longer needs precise order information (only upper bounds for the orders are required), and it can accurately estimate the channels subject to a scalar matrix ambiguity. Furthermore, a method is presented to resolve the scalar ambiguity matrix by using only one pilot OFDM block if the number of users is smaller than the length of an OFDM block. In general, when the total number of symbols in the transmitted pilot OFDM blocks is larger than the number of users, the ambiguity matrix can be resolved. Equalization methods are then presented based on the estimated channels. By using partial knowledge of the channels, a multipath subspace method is also proposed that reduces the computational complexity. Simulations show that the methods are effective and robust.

The rest of the paper is organized as follows. In Section II, we present the uplink MUMA-OFDM system model. The existence of the zero-forcing equalizer is discussed in Section III. Section IV is the main part of the paper, which develops the fundamental theory of the SS algorithm. The method to resolve the scalar ambiguity matrix through the use of one OFDM pilot block is considered in Section V. Equalization methods are discussed in Section VI. We give a multipath subspace algorithm in Section VII. Some simulation results and discussions are given in Section VIII. Finally, conclusions are drawn in Section IX.

Some notations are used throughout the paper. Superscripts $T, \dagger$, and $*$ stand for transpose, transconjugate, and conjugate, respectively. $\mathbf{I}_{q}$ is the identity matrix of order $q$, and $\otimes$ is the Kronecker product of matrices.

\section{MUMA-OFDM SYSTEM MODEL}

The multiuser and multiantenna OFDM system (MUMA-OFDM) was first proposed in [11], the uplink of which is shown in Fig. 1. Assume that there are $K$ users who share the same frequency band and $J$ omni-directional receiving antennas in the base station. Each antenna can receive signals from every user in the cell using the base station. The received signals from the $J$ antennas are sent to a central unit (CU) for processing. The goal of the processing is to recover the transmitted $K$ signals. We assume that each user uses ZP-OFDM [4], [15] instead of the CP-OFDM used in [11], because ZP-OFDM avoids IBI and, therefore, simplifies channel estimation and equalization [4], [15]. In zero-padding OFDM, the symbols to be transmitted are grouped into blocks with each block having $N$ symbols, each block is transformed by the inverse discrete Fourier transform (IDFT), and then, $L(L \leq N)$ zeros are added to the tail of each transformed block (zero-padding), where cyclic prefix is no longer needed. Each user transmits its OFDM modulated signal. Assume that all users are synchronized at block level. Let $\mathbf{s}_{i}^{(k)}$ be the block symbol to be transmitted by user $k$ at time $i$ (before OFDM modulation), where

$$
\begin{aligned}
& \mathbf{s}_{i}^{(k)}=\left(s_{i}^{(k)}(0), s_{i}^{(k)}(1), \cdots, s_{i}^{(k)}(N-1)\right)^{T} \\
& k=1,2, \cdots, K, \quad i=0,1, \cdots
\end{aligned}
$$

and its IDFT is $\mathbf{u}_{i}^{(k)} \cdot \mathbf{u}_{i}^{(k)}$ is zero-padded with $L$ zeros and then transmitted. Let $h^{(j, k)}(l)\left(l=0,1, \cdots, L_{j, k}\right)$ be the channel response (including the transmitting and receiving filters) from user $k$ to antenna $j$, where $L_{j, k}$ is the channel order $L_{j, k} \leq L$. Then, the received $i$ th block at antenna $j$ is

$$
\begin{gathered}
x_{i}^{(j)}(n)=\sum_{k=1}^{K} \sum_{l=0}^{L} h^{(j, k)}(l) u_{i}^{(k)}(n-l)+\boldsymbol{\eta}_{i}^{(j)}(n) \\
n=0,1, \cdots, M-1
\end{gathered}
$$


where $M=N+L, h^{(j, k)}(l)$ is zero-padded for $L_{j, k}+1 \leq l \leq$ $L$, and $\boldsymbol{\eta}_{i}^{(j)}(n)$ is the channel noise. Note that $u_{i}^{(k)}(n)=0$, if $n<0$ or $N \leq n<M$.

We assume that $J>K$. By defining

$$
\begin{aligned}
\mathbf{x}_{i}(n) & =\left(x_{i}^{(1)}(n), x_{i}^{(2)}(n), \cdots, x_{i}^{(J)}(n)\right)^{T} \\
\mathbf{h}^{(k)}(l) & =\left(h^{(1, k)}(l), h^{(2, k)}(l), \cdots, h^{(J, k)}(l)\right)^{T} \\
\boldsymbol{\eta}_{i}(n) & =\left(\boldsymbol{\eta}_{i}^{(1)}(n), \boldsymbol{\eta}_{i}^{(2)}(n), \cdots, \boldsymbol{\eta}_{i}^{(J)}(n)\right)^{T}
\end{aligned}
$$

we can express (1) into vector form as

$\mathbf{x}_{i}(n)=\sum_{k=1}^{K} \sum_{l=0}^{L} \mathbf{h}^{(k)}(l) u_{i}^{(k)}(n-l)+\eta_{i}(n), n=0,1, \cdots, M-1$.

By changing the order of the summation in (3) and defining

$$
\begin{aligned}
\mathbf{h}(l)= & \left(\mathbf{h}^{(1)}(l), \mathbf{h}^{(2)}(l), \cdots, \mathbf{h}^{(K)}(l)\right) \\
\mathbf{u}_{i}(n-l)= & {\left[\begin{array}{c}
u_{i}^{(1)}(n-l) \\
u_{i}^{(2)}(n-l) \\
\vdots \\
u_{i}^{(K)}(n-l)
\end{array}\right] }
\end{aligned}
$$

we can express (3) into

$$
\mathbf{x}_{i}(n)=\sum_{l=0}^{L} \mathbf{h}(l) \mathbf{u}_{i}(n-l)+\boldsymbol{\eta}_{i}(n), \quad n=0,1, \cdots, M-1 .
$$

Now let

$$
\begin{aligned}
& \mathbf{u}_{i}=\left[\begin{array}{c}
\mathbf{u}_{i}(0) \\
\mathbf{u}_{i}(1) \\
\vdots \\
\mathbf{u}_{i}(N-1)
\end{array}\right], \quad \mathbf{x}_{i}=\left[\begin{array}{c}
\mathbf{x}_{i}(0) \\
\mathbf{x}_{i}(1) \\
\vdots \\
\mathbf{x}_{i}(M-1)
\end{array}\right] \\
& \boldsymbol{\eta}_{i}=\left[\begin{array}{c}
\boldsymbol{\eta}_{i}(0) \\
\boldsymbol{\eta}_{i}(1) \\
\vdots \\
\boldsymbol{\eta}_{i}(M-1)
\end{array}\right] \\
& \mathbf{H}=\left[\begin{array}{ccccc}
\mathbf{h}(0) & & & & \\
\vdots & \mathbf{h}(0) & & & \\
\mathbf{h}(L) & \vdots & \ddots & & \\
& \mathbf{h}(L) & & \ddots & \\
& & \ddots & & \mathbf{h}(0) \\
& & & \ddots & \vdots \\
& & & & \mathbf{h}(L)
\end{array}\right]
\end{aligned}
$$

where $\mathbf{H}$ is a $J M \times K N$ block lower triangular Toeplitz matrix with the first block column being

$$
\left(\mathbf{h}^{T}(0), \mathbf{h}^{T}(1), \cdots, \mathbf{h}^{T}(L), 0, \cdots, 0\right)^{T} .
$$

Then, (6) is turned into

$$
\mathbf{x}_{i}=\mathbf{H u}_{i}+\boldsymbol{\eta}_{i}, \quad i=0,1, \cdots
$$

In a MUMA-SCZP system, each symbol block is zero-padded and transmitted. The only difference in the transmitting end between MUMA-OFDM and MUMA-SCZP is that the latter does not implement the IDFT on each symbol block. Therefore, the received signal in the MUMA-SCZP system is

$$
\mathbf{x}_{i}=\mathbf{H} \mathbf{s}_{i}+\boldsymbol{\eta}_{i}, \quad i=0,1, \cdots
$$

Hence, the channel estimation method for MUMA-OFDM is also valid for the MUMA-SCZP. In the receiving end, the equalization methods in the MUMA-OFDM and MUMA-SCZP have some differences, but all are realized by the FFT. This will be discussed in Section VI.

\section{EXISTENCE OF ZERO-FORCING EQUALIZER}

What we receive is the signal $\mathbf{x}_{i}$ with multiuser interference (MUI) and intersymbol interference (ISI) induced from the multipath effect. Is it possible to recover the transmitted signals $\mathbf{u}_{i}$ or $\mathbf{s}_{i}$, given that the signal-noise-ratio (SNR) is extremely high? Given that the noises can be ignored in (9), $\mathbf{u}_{i}$ is completely recoverable from $\mathbf{x}_{i}$ if and only if matrix $\mathbf{H}$ is of full column rank. From the structure of the matrix, we have the following theorem about the rank of matrix $\mathbf{H}$.

Theorem 1: If $\mathbf{h}(0)$ is of full column rank, then $\mathbf{H}$ is of full column rank.

Proof: If $\mathbf{h}(0)$ is of full column rank, then $\mathbf{h}^{T}(0)$ is of full row rank. Therefore, for any vector $\mathbf{c}$ of length $K$, the linear equation $\mathbf{h}^{T}(0) \mathbf{z}=\mathbf{c}$ always has a solution, which means that there exists a $J \times J$ matrix $\mathbf{D}_{l}$ such that

$$
\mathbf{h}^{T}(0) \mathbf{D}_{l}^{T}=-\mathbf{h}^{T}(l), \quad l=1,2, \cdots, L
$$

that is

$$
\mathbf{D}_{l} \mathbf{h}(0)=-\mathbf{h}(l), \quad l=1,2, \cdots, L
$$

Therefore, we can use a (block) elementary row matrix to multiply $\mathbf{H}$ on the left and turn it into a block diagonal matrix, that is, there exists an invertible $J M \times J M$ matrix $\mathbf{W}$ such that

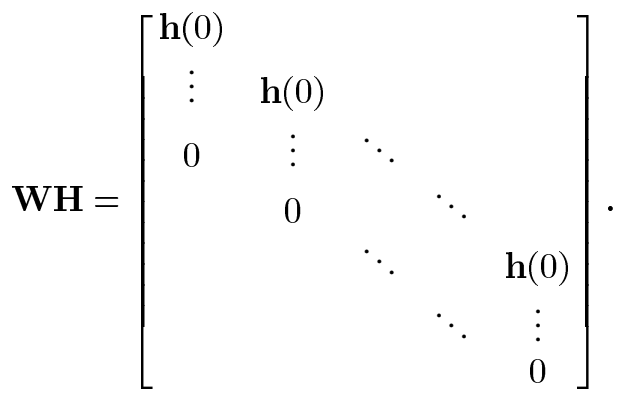

The matrix at the right is obviously of full column rank. Hence, $\mathbf{H}$ is of full column rank.

Noticing that $\mathbf{h}(0)$ is a $J \times K(J>K)$ tall matrix defined as

$$
\mathbf{h}(0)=\left[\begin{array}{ccccc}
h^{(1,1)}(0) & h^{(1,2)}(0) & \cdots & \cdots & h^{(1, K)}(0) \\
h^{(2,1)}(0) & h^{(2,2)}(0) & \cdots & \cdots & h^{(2, K)}(0) \\
\vdots & \vdots & & & \vdots \\
h^{(J, 1)}(0) & h^{(J, 2)}(0) & \cdots & \cdots & h^{(J, K)}(0)
\end{array}\right]
$$


we can see that the full column rank property of $\mathbf{h}(0)$ is almost surely guaranteed because signal propagation from each of the $K$ users scattered in the cell is most likely independent. In the following, we assume that this condition holds. Even if $\mathbf{h}(0)$ is not of full column rank, it is still possible that $\mathbf{H}$ is of full column rank.

If the channel responses are known, we can use the joint detection method proposed in [11] or other means to recover the transmitted symbols, which will be discussed in Section VI. Unfortunately, channel estimation in MIMO system is usually a very difficult task, and [11] does not discuss the problem. We will address the problem in the following.

\section{SUBSPACE AlgORITHM}

In this section, we consider the blind identification of channels by using only the SOS of the received signal samples. Before discussing the algorithm, we make the following assumptions for the statistical properties of transmitted symbols $s_{l}^{(k)}(n)$ and channel noise $\eta_{i}^{(j)}(n)$.

A1) Noises are white and uncorrelated, that is

$$
\mathrm{E}\left(\boldsymbol{\eta}_{i}^{(j)}(n)\left(\eta_{l}^{(k)}(m)\right)^{*}\right)= \begin{cases}\sigma_{\eta}^{2}, & (i, j, n)=(l, k, m) \\ 0, & (i, j, n) \neq(l, k, m) .\end{cases}
$$

A2) Noises and transmitted signals are uncorrelated, that is, $\mathrm{E}\left(\boldsymbol{\eta}_{i}^{(j)}(n)\left(s_{l}^{(k)}(m)\right)^{*}\right)=0$. Here, $\mathrm{E}(y)$ means the mathematical expectation of a random variable $y$.

\section{A. Theoretical Development}

Now, we consider the statistical auto-correlation matrices of $\mathbf{x}_{i}$. Based on assumptions A1)and A2), we can verify that

$$
\mathbf{R}_{x}=\mathrm{E}\left(\mathbf{x}_{i} \mathbf{x}_{i}^{\dagger}\right)=\mathbf{H R}_{u} \mathbf{H}^{\dagger}+\sigma_{\eta}^{2} \mathbf{I}_{M J}
$$

where $\mathbf{R}_{u}=\mathrm{E}\left(\mathbf{u}_{i} \mathbf{u}_{i}^{\dagger}\right)$ is a positive definite matrix.

The smallest eigenvalue of matrix $\mathbf{R}_{x}$ is $\sigma_{\eta}^{2}$. Since the rank of $\mathbf{H R}_{u} \mathbf{H}^{\dagger}$ is $N K$, there are $q=M J-N K$ co-orthogonal eigenvectors corresponding to the smallest eigenvalue. These eigenvectors are denoted by $\boldsymbol{\beta}_{i}(i=0,1, \cdots, q-1)$. Based on a simple mathematical derivation used in the standard subspace method [19], [28], we know that

$$
\boldsymbol{\beta}_{i}^{\dagger} \mathbf{H}=0, \quad i=0,1, \cdots, q-1
$$

that is, $\boldsymbol{\beta}_{i}(i=0,1, \cdots, q-1)$ spans the left null space of $\mathbf{H}$. Having known the left null space, we can determine the range space, which is denoted by $\operatorname{span}(\mathbf{H})$, which is all possible linear combinations of the column vectors of $\mathbf{H}$. Equivalently, we can also treat (13) as $q K N$ linear equations with $h^{(j, k)}(l)$ as unknowns. For $J \geq K$ and $N \geq L$, the number of equations is usually larger than that of the unknowns. However, since many of the linear equations may be dependent, it is hard to say if the unknowns can be determined. In general, knowing $\operatorname{span}(\mathbf{H})$ cannot determine matrix $\mathbf{H}$. However, for some matrix with special structure, it is possible to determine $\mathbf{H}$ by $\operatorname{span}(\mathbf{H})$ up to a certain ambiguity. The matrix $\mathbf{H}$ here is a block lower triangular Toeplitz full column rank matrix. The following theorem states that $\mathbf{H}$ is uniquely determined by $\operatorname{span}(\mathbf{H})$ subject to a $K \times K$ matrix ambiguity.
Theorem 2: Let

$$
\begin{aligned}
\mathbf{H} & =\left[\begin{array}{ccccc}
\mathbf{h}(0) & & & & \\
\vdots & \mathbf{h}(0) & & & \\
\mathbf{h}(L) & \vdots & \ddots & & \\
& \mathbf{h}(L) & & \ddots & \\
& & \ddots & & \mathbf{h}(0) \\
& & & \ddots & \vdots \\
& & & & \mathbf{h}(L)
\end{array}\right] \\
\hat{\mathbf{H}} & =\left[\begin{array}{ccccc}
\hat{\mathbf{h}}(0) & & & & \\
\vdots & \hat{\mathbf{h}}(0) & & & \\
\hat{\mathbf{h}}(L) & \vdots & \ddots & & \\
& \hat{\mathbf{h}}(L) & & \ddots & \\
& & \ddots & & \hat{\mathbf{h}}(0) \\
& & & \ddots & \vdots \\
& & & & \hat{\mathbf{h}}(L)
\end{array}\right]
\end{aligned}
$$

where $\mathbf{h}(l)$ and $\hat{\mathbf{h}}(l)(l=0,1, \cdots, L)$ are $J \times K$ matrices, and $\mathbf{h}(0)$ and $\mathbf{h}(0)$ are of full column rank. If $\operatorname{span}(\mathbf{H})=\operatorname{span}(\hat{\mathbf{H}})$, there exists a $K \times K$ invertible matrix b such that

$$
\hat{\mathbf{h}}(l)=\mathbf{h}(l) \mathbf{b}, \quad l=0,1, \cdots, L .
$$

Proof: It is easy to verify that $\operatorname{span}(\mathbf{H})=\operatorname{span}(\hat{\mathbf{H}})$ if and only if there exists a $K N \times K N$ invertible matrix $\mathbf{B}$ such that

$$
\hat{\mathbf{H}}=\mathbf{H B} \text {. }
$$

We section $\mathbf{B}$ into $K \times K$ blocks and express it as

$$
\mathbf{B}=\left[\begin{array}{ccccc}
\mathbf{B}_{11} & \mathbf{B}_{12} & \cdots & \cdots & \mathbf{B}_{1 N} \\
\mathbf{B}_{21} & \mathbf{B}_{22} & \cdots & \cdots & \mathbf{B}_{2 N} \\
\vdots & \vdots & & & \vdots \\
\mathbf{B}_{N 1} & \mathbf{B}_{N 2} & \cdots & \cdots & \mathbf{B}_{N N}
\end{array}\right]
$$

where $\mathbf{B}_{i j}$ are $K \times K$ matrices.

First, we want to prove that $\mathbf{B}$ must be a block lower triangular matrix and that all its diagonal blocks are the same. We use mathematical induction. The $i$ th row of $\hat{\mathbf{H}}$ is the multiplication of the $i$ th row of $\mathbf{H}$ and $\mathbf{B}$. Considering the first row, we have

$$
\mathbf{h}(0) \mathbf{B}_{11}=\hat{\mathbf{h}}(0), \quad \mathbf{h}(0) \mathbf{B}_{1 j}=0, \quad j=2, \cdots, N .
$$

Since $\mathbf{h}(0)$ and $\hat{\mathbf{h}}(0)$ are of full column rank, we know that $\mathbf{B}_{11}$ must also be of full rank, that is, $\mathbf{B}_{11}$ is invertible. In addition, $\mathbf{B}_{1 j}=0$, and $j=2, \cdots, N$.

Now, assuming that $\mathbf{B}_{i j}=0$ and $\mathbf{B}_{i i}=\mathbf{B}_{11}$, where $i=$ $1, \cdots, k$, and $j \geq i+1$, we want to show that $\mathbf{B}_{k+1, j}=0(j \geq$ $k+2$ ), and $\mathbf{B}_{k+1, k+1}=\mathbf{B}_{11}$. Considering the $(k+1)$ th row, based on the assumption, we have

$$
\mathbf{h}(0) \mathbf{B}_{k+1, k+1}=\hat{\mathbf{h}}(0), \mathbf{h}(0) \mathbf{B}_{k+1, j}=0, j=k+2, \cdots, N \text {. }
$$

Since we also have $\mathbf{h}(0) \mathbf{B}_{11}=\hat{\mathbf{h}}(0)$, it is obvious that $\mathbf{B}_{k+1, k+1}=\mathbf{B}_{11}$. Furthermore, the full column rank property of $\mathbf{h}(0)$ means that $\mathbf{B}_{k+1, j}=0$, and $j=k+2, \cdots, N$. Based 
on the mathematical induction law, we know that $\mathbf{B}$ is a block lower triangular matrix with identical diagonal blocks.

Second, we prove that $\hat{\mathbf{h}}(l)=\mathbf{h}(l) \mathbf{b}(l=0,1, \cdots, L)$ with some $\mathbf{b}$. For simplicity, we use $\mathbf{b}$ to denote the matrix $\mathbf{B}_{11}$. It is shown above that $\mathbf{B}$ is a block lower triangular matrix and that all its diagonal blocks are b. Considering the last column of $\hat{\mathbf{H}}$, we see that

$$
\left[\begin{array}{c}
0 \\
\vdots \\
0 \\
\hat{\mathbf{h}}(0) \\
\vdots \\
\hat{\mathbf{h}}(L)
\end{array}\right]=\mathbf{H}\left[\begin{array}{c}
0 \\
\vdots \\
0 \\
\mathbf{b}
\end{array}\right]=\left[\begin{array}{c}
0 \\
\vdots \\
0 \\
\mathbf{h}(0) \mathbf{b} \\
\vdots \\
\mathbf{h}(L) \mathbf{b}
\end{array}\right] .
$$

Therefore, we finally get

$$
\hat{\mathbf{h}}(l)=\mathbf{h}(l) \mathbf{b}, \quad l=0,1, \cdots, L .
$$

From this theorem, we see that the two restrictions on the SS method for general MIMO system [19], that is, the requirement of exact channel order and the polynomial matrix ambiguity, are lifted, thanks to the ZP-OFDM structure. Sensitivity to channel order overestimation is also a major problem for many known SS methods in a SIMO system [28], [34].

\section{B. Implementation of the Subspace Method}

Equation (13) can be expressed equivalently as

$$
\mathbf{H}^{\dagger} \boldsymbol{\beta}_{i}=0, \quad i=0,1, \cdots, q-1 .
$$

By dividing the vector $\boldsymbol{\beta}_{i}$ into blocks as

$$
\boldsymbol{\beta}_{i}=\left(\boldsymbol{\beta}_{i}^{T}(M-1), \boldsymbol{\beta}_{i}^{T}(M-2), \cdots, \boldsymbol{\beta}_{i}^{T}(0)\right)^{T}
$$

where $\boldsymbol{\beta}_{i}(m)(m=0,1, \cdots, M-1)$ are $J \times 1$ vectors, it is easy to turn (18) into

$$
\sum_{l=0}^{L} \mathbf{h}^{\dagger}(l) \boldsymbol{\beta}_{i}(n+L-l)=0, \quad n=0,1, \cdots, N-1
$$

or, equivalently

$$
\sum_{l=0}^{L} \boldsymbol{\beta}_{i}^{\dagger}(n+L-l) \mathbf{h}(l)=0, \quad n=0,1, \cdots, N-1 .
$$

Denote two matrices $\mathbf{G}_{i}$ and $\overline{\mathbf{H}}$ as

$$
\begin{aligned}
\mathbf{G}_{i} & =\left[\begin{array}{cccc}
\boldsymbol{\beta}_{i}^{\dagger}(L) & \boldsymbol{\beta}_{i}^{\dagger}(L-1) & \cdots & \boldsymbol{\beta}_{i}^{\dagger}(0) \\
\boldsymbol{\beta}_{i}^{\dagger}(L+1) & \boldsymbol{\beta}_{i}^{\dagger}(L) & \cdots & \boldsymbol{\beta}_{i}^{\dagger}(1) \\
\vdots & \vdots & & \vdots \\
\boldsymbol{\beta}_{i}^{\dagger}(M-1) & \boldsymbol{\beta}_{i}^{\dagger}(M-2) & \cdots & \boldsymbol{\beta}_{i}^{\dagger}(N-1)
\end{array}\right] \\
\overline{\mathbf{H}} & =\left[\begin{array}{c}
\mathbf{h}(0) \\
\mathbf{h}(1) \\
\vdots \\
\mathbf{h}(L)
\end{array}\right] .
\end{aligned}
$$

Then, (21) is equivalent to

$$
\mathbf{G}_{i} \overline{\mathbf{H}}=0, \quad i=0,1, \cdots, q-1
$$

which can also be expressed as

$$
\mathbf{G} \overline{\mathbf{H}}=0
$$

where $\mathbf{G}$ is a $q N \times J(L+1)$ matrix defined as

$$
\mathbf{G}=\left[\begin{array}{c}
\mathbf{G}_{0} \\
\mathbf{G}_{1} \\
\vdots \\
\mathbf{G}_{q-1}
\end{array}\right] .
$$

Based on Matlab notation, we use $\overline{\mathbf{H}}(:, j)$ to denote the $j$ th column of the matrix $\overline{\mathbf{H}}$. Then

$$
\mathbf{G} \overline{\mathbf{H}}(:, j)=0, \quad j=1,2, \cdots, K
$$

that is, $\mathbf{\mathbf { H }}(:, j)$ belongs to the right null space of $\mathbf{G}$. From Theorem 2, it is easy to show that the dimension of right null space of $\mathbf{G}$ is $K$. Therefore, $\overline{\mathbf{H}}(:, j)(j=1,2, \cdots, K)$ is actually a basis of the null space. Therefore, matrix $\overline{\mathbf{H}}$ can be obtained by finding a basis for the null space, which can be achieved by solving the eigenvectors of eigenvalue 0 for matrix $\mathbf{G}$ or singular value decomposition (SVD). In practice, due to errors in computing the auto-correlation matrix and roundoff errors, (26) will not hold precisely, and therefore, we should choose $K$ right singular vectors corresponding to the $K$ least singular values, respectively, to form the columns of $\overline{\mathbf{H}}$.

The channel responses can also be determined by minimizing a target function. Let

$$
\epsilon(\overline{\mathbf{H}})=\sum_{i=0}^{q-1}\left\|\mathbf{G}_{i} \overline{\mathbf{H}}\right\|_{F}^{2}
$$

where $\|\cdot\|_{F}$ means the Frobenius norm for matrix. Obviously, to find the channel responses is to minimize the function $\epsilon(\overline{\mathbf{H}})$. In order to avoid trivial (zero) solutions, a constraint must be imposed, for example, $\|\overline{\mathbf{H}}\|_{F}=1$.

As stated above, the solution is still not unique. We should choose a solution $\overline{\mathbf{H}}_{0}$ such that $\mathbf{h}(0)$ is invertible. The actual channel response matrix is then

$$
\overline{\mathbf{H}}=\overline{\mathbf{H}}_{0} \mathbf{b}
$$

where $\mathbf{b}$ is a $K \times K$ invertible matrix to be determined.

The subspace method is summarized as follows.

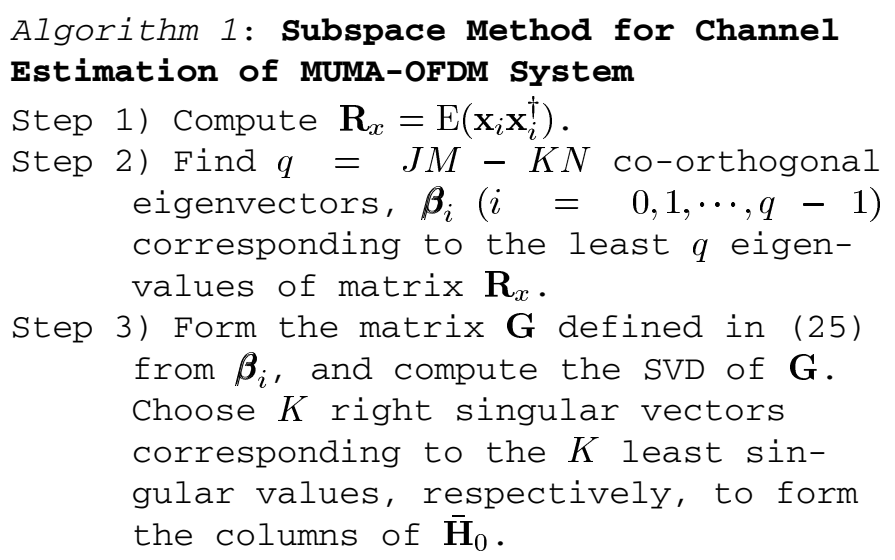


Step 4) The channel matrix is $\overline{\mathbf{H}}=\overline{\mathbf{H}}_{0} \mathbf{b}$, where $\mathbf{b}$ is a $K \times K$ invertible matrix to be determined.

\section{Determination of the Number of Users}

For most communication applications, the number of users $K$ is known a priori at the base station, and therefore, the SS method is easy to implement. If $K$ is unknown, we need to estimate it first. A method for finding $K$ is described in the following. If the matrix $\mathbf{R}_{x}$ is computed sufficient accurately from the received signal samples, we know that the smallest eigenvalue of matrix $\mathbf{R}_{x}$ is $\sigma_{\eta}^{2}$, and there are $q=M J-N K$ co-orthogonal eigenvectors corresponding to the eigenvalue. Therefore, by computing the eigenvalues of $\mathbf{R}_{x}$, we can find the number $q$ and, hence, get $K$ by $K=(M J-q) / N$. However, in practice, the auto-correlation matrix $\mathbf{R}_{x}$ can only be computed from some finite number of received signal samples and, therefore, usually is not accurate enough. The most commonly used method for the computation is

$$
\mathbf{R}_{x}=\mathrm{E}\left(\mathbf{x}_{i} \mathbf{x}_{i}^{\dagger}\right) \approx \frac{1}{L_{s}} \sum_{i=0}^{L_{s}-1} \mathbf{x}_{i} \mathbf{x}_{i}^{\dagger}
$$

where $L_{s}$ is the number of block samples used. Due to statistical and roundoff errors, the smallest eigenvalue of $\mathbf{R}_{x}$ is spread into a number of different eigenvalues that are approximately equal. Therefore, it may be difficult to determine the number $q$, especially when the received SNR is low. However, if we have some knowledge on the noise level and have obtained an estimation, say, $\hat{\sigma}_{\eta}^{2}$, of the noise variance, we can use it as a threshold to determine $q$. If an eigenvalue is smaller than a small (say, 2) multiples of the threshold, it is treated as a smallest eigenvalue. An estimation of $q$, say, $\hat{q}$, is hence obtained. Based on $\hat{q}, K$ is therefore estimated by $\hat{K}=(M J-\hat{q}) / N . K$ is chosen to be the nearest integer of $\hat{K}$. If $\hat{q}=q+\delta$, we have $\hat{K}=K-\delta / N$. If $|\delta|<N / 2$, the nearest integer of $\hat{K}$ is $K$. We can also use other means such as the method in [35] to estimate the number $q$ (and hence $K$ ).

\section{Resolve the MATRIX Ambiguity}

Let $\mathbf{H}_{0}$ be the estimated channel matrix in the form as (8). From Theorem 2, we know that the actual channel matrix should be

$$
\mathbf{H}=\mathbf{H}_{0} \operatorname{diag}(\mathbf{b}, \mathbf{b}, \cdots, \mathbf{b})
$$

where $\mathbf{b}$ is the ambiguity matrix to be determined. Therefore

$$
\mathbf{x}_{i}=\mathbf{H u}_{i}+\boldsymbol{\eta}_{i}=\mathbf{H}_{0} \operatorname{diag}(\mathbf{b}, \mathbf{b}, \cdots, \mathbf{b}) \mathbf{u}_{i}+\boldsymbol{\eta}_{i} .
$$

Since $\mathbf{H}_{0}$ is of full column rank, we can find a $K N \times K N$ matrix $\mathbf{V}=\left(\mathbf{H}_{0}^{\dagger} \mathbf{H}_{0}\right)^{-1} \mathbf{H}_{0}^{\dagger}$. Let $\mathbf{y}_{i}=\mathbf{V} \mathbf{x}_{i}$ and $\xi_{i}=\mathbf{V} \boldsymbol{\eta}_{i}$. Then

$$
\mathbf{y}_{i}=\operatorname{diag}(\mathbf{b}, \mathbf{b}, \cdots, \mathbf{b}) \mathbf{u}_{i}+\xi_{i} .
$$

By dividing the vector $\mathbf{y}_{i}$ and $\xi_{i}$ into blocks as

$$
\begin{aligned}
\mathbf{y}_{i} & =\left(\mathbf{y}_{i}^{T}(0), \mathbf{y}_{i}^{T}(1), \cdots, \mathbf{y}_{i}^{T}(N-1)\right)^{T} \\
\xi_{i} & =\left(\xi_{i}^{T}(0), \xi_{i}^{T}(1), \cdots, \xi_{i}^{T}(N-1)\right)^{T}
\end{aligned}
$$

where $\mathbf{y}_{i}(n)$ and $\xi_{i}(n)(n=0,1, \cdots, N-1)$ are $K \times 1$ vectors, according to the definition of $\mathbf{u}_{i}$ in (7), we get

$$
\mathbf{y}_{i}(n)=\mathbf{b u}_{i}(n)+\xi_{i}(n), \quad n=0,1, \cdots, N-1 .
$$

Let

$$
\begin{aligned}
\mathbf{Y}_{i} & =\left(\mathbf{y}_{i}(0), \mathbf{y}_{i}(1), \cdots, \mathbf{y}_{i}(N-1)\right) \\
\mathbf{U}_{i} & =\left(\mathbf{u}_{i}(0), \mathbf{u}_{i}(1), \cdots, \mathbf{u}_{i}(N-1)\right) \\
\boldsymbol{\Gamma}_{\mathbf{i}} & =\left(\xi_{i}(0), \xi_{i}(1), \cdots, \xi_{i}(N-1)\right) .
\end{aligned}
$$

Then

$$
\mathbf{Y}_{i}=\mathbf{b U}_{i}+\Gamma_{\mathbf{i}}
$$

If a pilot OFDM block is sent and $N \geq K, \mathbf{U}_{i}$ can be assumed to be full row rank. Then, we can solve $\mathbf{b}$ by

$$
\mathbf{b} \approx \mathbf{Y}_{i} \mathbf{U}_{i}^{\dagger}\left(\mathbf{U}_{i} \mathbf{U}_{i}^{\dagger}\right)^{-1}
$$

If $N<K$, more pilot blocks are needed. In general, when the total number of symbols in the transmitted pilot OFDM blocks is larger than the number of users, the ambiguity matrix can be resolved by the same method.

\section{EQUALIZATION}

From (34), equalization is easily achieved by

$$
\mathbf{u}_{i}(n) \approx \mathbf{b}^{-1} \mathbf{y}_{i}(n), \quad n=0,1, \cdots, N-1 .
$$

Then, the transmitted symbols are obtained by

$$
\mathbf{s}_{i}^{(k)}=\operatorname{DFT}\left(\mathbf{u}_{i}^{(k)}\right), \quad k=1,2, \cdots, K .
$$

We call this method "time domain equalization" (TDE). A major advantage of OFDM is that it can be equalized in the frequency domain by using FFT. This advantage is kept in the ZP-OFDM. Unlike in the CP-OFDM, where the first $L$ elements of each received block are discarded, in ZP-OFDM, the received signal is first overlap added before the DFT is implemented. The "frequency domain equalization" (FDE) for the MUMA-OFDM system is discussed in the following.

Each received block is overlap added as

$$
\hat{x}_{i}^{(j)}(n)= \begin{cases}x_{i}^{(j)}(n)+x_{i}^{(j)}(n+N), & n=0, \cdots, L-1 \\ x_{i}^{(j)}(n), & n=L, \cdots, N-1 .\end{cases}
$$

Each overlap added block is then transformed by the DFT. Let $\tilde{\mathbf{x}}_{i}^{(j)}=\operatorname{DFT}\left(\hat{\mathbf{x}}_{i}^{(j)}\right)$. Zero-pad each channel $\mathbf{h}^{(j, k)}$ to length $N$ and let the DFT (length- $N$ ) of the channel be $\tilde{\mathbf{h}}^{(j, k)}$, that is, $\tilde{\mathbf{h}}^{(j, k)}=\operatorname{DFT}\left(\mathbf{h}^{(j, k)}\right)$. Then

$\tilde{x}_{i}^{(j)}(n)=\sum_{k=1}^{K} \tilde{h}^{(j, k)}(n) s_{i}^{(k)}(n)+\zeta_{i}^{(j)}(n), n=0,1, \cdots, N-1$

where $\zeta_{i}^{(j)}(n)$ is the noise after the DFT. Let

$$
\begin{aligned}
\tilde{\mathbf{x}}_{i}(n) & =\left(\tilde{x}_{i}^{(1)}(n), \cdots, \tilde{x}_{i}^{(J)}(n)\right)^{T} \\
\mathbf{s}_{i}(n) & =\left(s_{i}^{(1)}(n), \cdots, s_{i}^{(K)}(n)\right)^{T}
\end{aligned}
$$




$$
\begin{aligned}
\zeta_{i}(n) & =\left(\zeta_{i}^{(1)}(n), \cdots, \zeta_{i}^{(J)}(n)\right)^{T} \\
\tilde{\mathbf{h}}(n) & =\left[\begin{array}{ccc}
\tilde{h}^{(1,1)}(n) & \cdots & \tilde{h}^{(1, K)}(n) \\
\tilde{h}^{(2,1)}(n) & \cdots & \tilde{h}^{(2, K)}(n) \\
\vdots & \cdots & \vdots \\
\tilde{h}^{(J, 1)}(n) & \cdots & \tilde{h}^{(J, K)}(n)
\end{array}\right] .
\end{aligned}
$$

Then, (41) is expressed as

$$
\tilde{\mathbf{x}}_{i}(n)=\tilde{\mathbf{h}}(n) \mathbf{s}_{i}(n)+\zeta_{i}(n), \quad n=0,1, \cdots, N-1 .
$$

Therefore, a ZF equalizer can be constructed as

$\mathbf{s}_{i}(n)=\left(\tilde{\mathbf{h}}^{\dagger}(n) \tilde{\mathbf{h}}(n)\right)^{-1} \tilde{\mathbf{h}}^{\dagger}(n) \tilde{\mathbf{x}}_{i}(n), \quad n=0,1, \cdots, N-1$

which is the same as the joint detection method in [11] for MUMA-OFDM (with cyclic prefix).

The advantage of FDE over TDE is that it reduces the computational complexity. For slow time fading channels, where the channel is assumed to remain unchanged within several OFDM blocks, the matrix $\mathbf{V}$ in TDE and $\left(\tilde{\mathbf{h}}^{\dagger}(n) \tilde{\mathbf{h}}(n)\right)^{-1}$ in FDE are constants in several OFDM blocks and therefore can be computed once in several blocks. Therefore, we ignore the computation of these matrices when considering the computational complexities of equalizing one block. To recover one symbol block, TDE and FDE use $\mathrm{O}\left(K^{2} N^{2}\right)$ and $\mathrm{O}(J K N \log N)$ operations (multiplications and additions), respectively. If $J$ is not much larger than $K$ and $N$ is large, the reduction by FDE is substantial. If we make full use of the property that each channel $\mathbf{h}^{(j, k)}$ is zero-padded before the DFT, the computational complexity of the FDE can be further reduced if $L$ is much smaller than $N$.

If the system is a MUMA-SCZP, the IDFT must be implemented on the output of (47) to get the transmitted symbols. Therefore, the overall computational complexity increases by $K$ FFT operations compared with that of MUMA-OFDM.

\section{Multipath SubSPACE Method}

The channel $h$ described above includes not only the multipath effect but also the transmitting and receiving filters. Only the multipath effect is unknown, and so, as in [22], we can simplify the SS method. Assume that the multipath channel response from user $k$ to antenna $j$ is $f^{(j, k)}(l)(l=0,1, \cdots, r)$, where $r$ is an upper bound for all the channel orders. Then, the compound channel response $h^{(j, k)}(l)$ is the convolution of the multipath, the transmitting, and the receiving channel responses, and it can be expressed by [22]

$$
\begin{aligned}
{\left[\begin{array}{c}
h^{(j, k)}(0) \\
h^{(j, k)}(1) \\
\vdots \\
h^{(j, k)}(L)
\end{array}\right] } & =\mathbf{A}\left[\begin{array}{c}
f^{(j, k)}(0) \\
f^{(j, k)}(1) \\
\vdots \\
f^{(j, k)}(r)
\end{array}\right] \\
j & =1,2, \cdots, J, \quad k=1,2, \cdots, K
\end{aligned}
$$

where $\mathbf{A}$ is a known $(L+1) \times(r+1)$ matrix that includes the transmitting and receiving filters. Typically, $L$ is much larger than $r$. Similar to the construction of $\overline{\mathbf{H}}$, we can construct the multipath channel response matrix as

$$
\overline{\mathbf{F}}=\left[\begin{array}{c}
\mathbf{f}(0) \\
\mathbf{f}(1) \\
\vdots \\
\mathbf{f}(r)
\end{array}\right]
$$

where

$$
\mathbf{f}(m)=\left[\begin{array}{cccc}
f^{(1,1)}(m) & f^{(1,2)}(m) & \cdots & f^{(1, K)}(m) \\
f^{(2,1)}(m) & f^{(2,2)}(m) & \cdots & f^{(2, K)}(m) \\
\vdots & \vdots & & \vdots \\
f^{(J, 1)}(m) & f^{(J, 2)}(m) & \cdots & f^{(J, K)}(m)
\end{array}\right]
$$

Based on (48), it can be proved that

$$
\overline{\mathbf{H}}=\left(\mathbf{A} \otimes \mathbf{I}_{J}\right) \overline{\mathbf{F}} .
$$

Let $\hat{\mathbf{G}}=\mathbf{G}\left(\mathbf{A} \otimes \mathbf{I}_{J}\right)$; then, (24) is turned into

$$
\hat{\mathbf{G}} \overline{\mathbf{F}}=0 .
$$

The columns of $\overline{\mathbf{F}}$ are obtained directly from the right null space of $\hat{\mathbf{G}}$. Noticing that the size of $\hat{\mathbf{G}}$ is $q N \times J(r+1)$, which is much smaller than that of $\mathbf{G}$, we know that the computational complexity for (52) is much lower than that for (24), which is the major advantage of the multipath SS method.

$\overline{\mathbf{F}}$ can also be found by minimizing the function $\hat{\epsilon}(\overline{\mathbf{F}})=\sum_{i=0}^{q-1}\left\|\mathbf{G}_{i}\left(\mathbf{A} \otimes \mathbf{I}_{J}\right) \overline{\mathbf{F}}\right\|_{F}^{2}$ under the condition $\|\overline{\mathbf{F}}\|_{F}=1$, where the target function has only $J K(r+1)$ other than $J K(L+1)$ unknowns.

\section{SIMULATIONS AND DISCUSSIONS}

\section{A. Simulations}

The auto-correlation matrix $\mathbf{R}_{x}$ is computed by (29). In the following, SNR means the ratio of the average received signal power with the average noise power as

$$
\mathrm{SNR}=\frac{\mathrm{E}\left(\left\|\mathbf{x}_{i}(n)-\boldsymbol{\eta}_{i}(n)\right\|^{2}\right)}{\mathrm{E}\left(\left\|\boldsymbol{\eta}_{i}(n)\right\|^{2}\right)} .
$$

Simulations show that the methods are very effective. Some examples are given below.

Example 1-Determination of the Number of Users: In a two-user, three-antenna MIMO system, the true channel orders are less than 10, the transmitted baseband signals are 16-QAM, and the length of each OFDM block is $N=32$, which is zero-padded to block of length $M=41$. The number of received blocks for computing the matrix $\mathbf{R}_{x}$ is 150 . In Fig. 2, the first 70 smallest eigenvalues are given for three different noise levels with corresponding $\sigma_{\eta}^{2}$ being $6.1946,0.4405$, and 0.0313 , respectively. Theoretically, there should be 59 smallest eigenvalues equal to $\sigma_{\eta}^{2}$, but numerical results are obviously not the case. The smallest eigenvalue is spread into a number of different eigenvalues. For high SNR, the first 59 eigenvalues are obviously much smaller than the 60th eigenvalue. Therefore, we have decided that $q=59$ and then found that $K=$ 


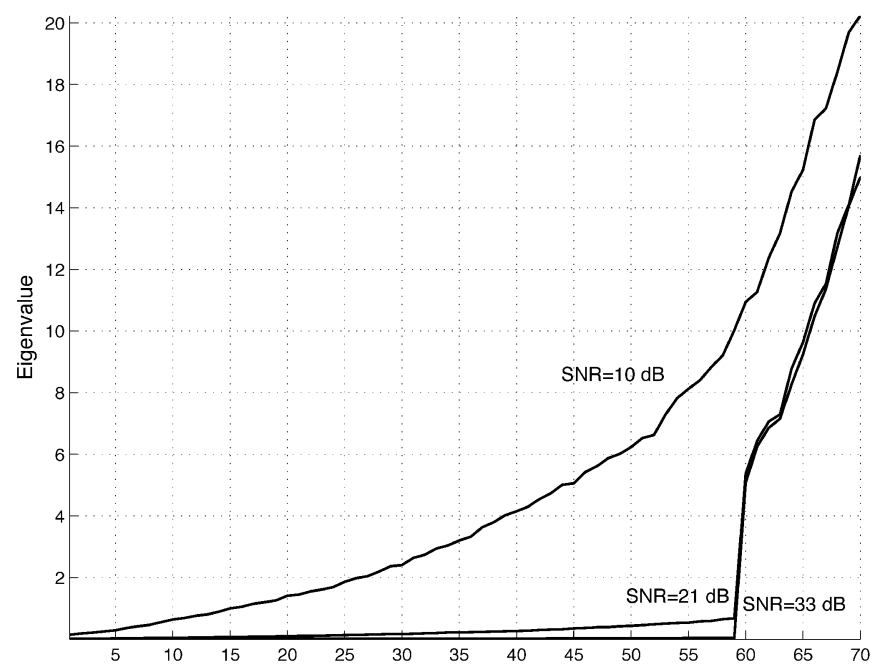

Fig. 2. Eigenvalues of the autocorrelation matrix.

$(41 \cdot 3-59) / 32=2$. Even if a slightly wrong decision is made, for example, $q=56$, we find $K=2.1$, and the nearest integer is 2 . In the case of SNR $=10$, there is no sharp difference between the first 59 eigenvalues and the 60th eigenvalue. If we have an estimation of $\sigma_{\eta}^{2}$ as $\hat{\sigma}_{\eta}^{2}=6$, the number of eigenvalues smaller than $2 \hat{\sigma}_{\eta}^{2}$ is 61 . Therefore, we have decided that $q=61$, and hence, $K=1.9$. We can still obtain the correct answer that $K=2$.

Example 2-Channel Estimation: The system parameters are the same as those in the last example. In order to resolve the ambiguity matrix, we assume that the first transmitted OFDM block is a pilot. Based on this known block, the ambiguity matrix is solved by (37). The normalized root mean square error (RMSE) between the estimated and true channel responses is defined as

$$
\operatorname{RMSE}=\sqrt{\frac{\sum_{j=1}^{J} \sum_{k=1}^{K}\left\|\hat{\mathbf{h}}^{(j, k)}-\mathbf{h}^{(j, k)}\right\|^{2}}{\sum_{j=1}^{J} \sum_{k=1}^{K}\left\|\mathbf{h}^{(j, k)}\right\|^{2}}}
$$

where $\hat{\mathbf{h}}^{(j, k)}$ and $\mathbf{h}^{(j, k)}$ are the estimated and true channel responses, respectively. Fig. 3 shows the RMSE. The amplitudes of the channel responses are shown in Figs. 4 ( $\mathrm{SNR}=21.4 \mathrm{~dB}$ ) and $5(\mathrm{SNR}=28.5 \mathrm{~dB})$, respectively, where dashed lines are for estimated channels and solid lines for true channels. The results are averaged over 50 Monte Carlo realizations.

Example 3-Equalization: After obtaining the estimated channel responses, TDE and FDE, which was discussed above, can be used for equalization. For comparison, we also test the FDE method, assuming the true channel responses are known. Simulations show that TDE and FDE do not have much difference in accuracy. When the SNR is high, the channels can be estimated very accurately, and therefore, the two methods approach the FDE with true channel responses. In Fig. 6 and 7 , the bit error rates (BERs) are shown as the SNR varies from 5 to $40 \mathrm{~dB}$, where lines with marks $\circ, \triangle$, and $\nabla$ are for FDEs with true channel responses, TDEs and FDEs with estimated channel responses, respectively, and the true channel is shown in Fig. 4. The symbol constellation are BPSK and 16-QAM, respectively, and the block length $N$ is 32 . The BER is obtained

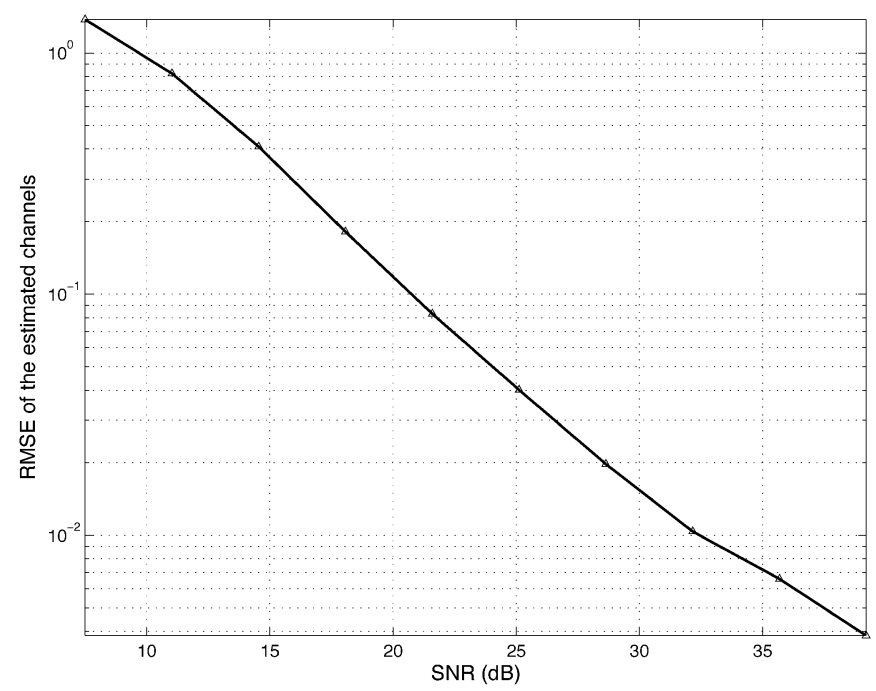

Fig. 3. RMSE of the estimated composite channel responses.

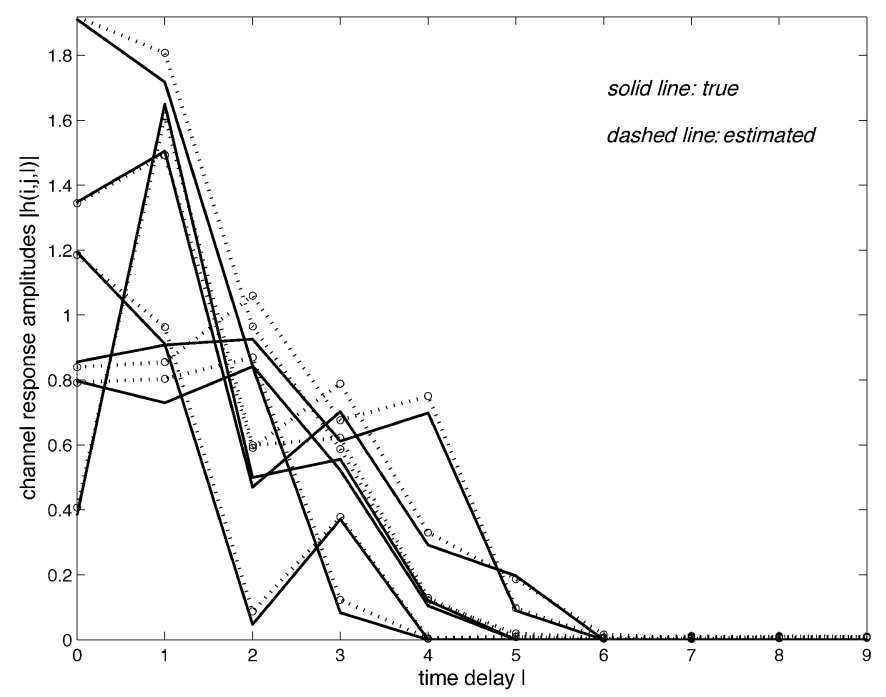

Fig. 4. Channel response amplitudes $(\mathrm{SNR}=21.4 \mathrm{~dB})$.

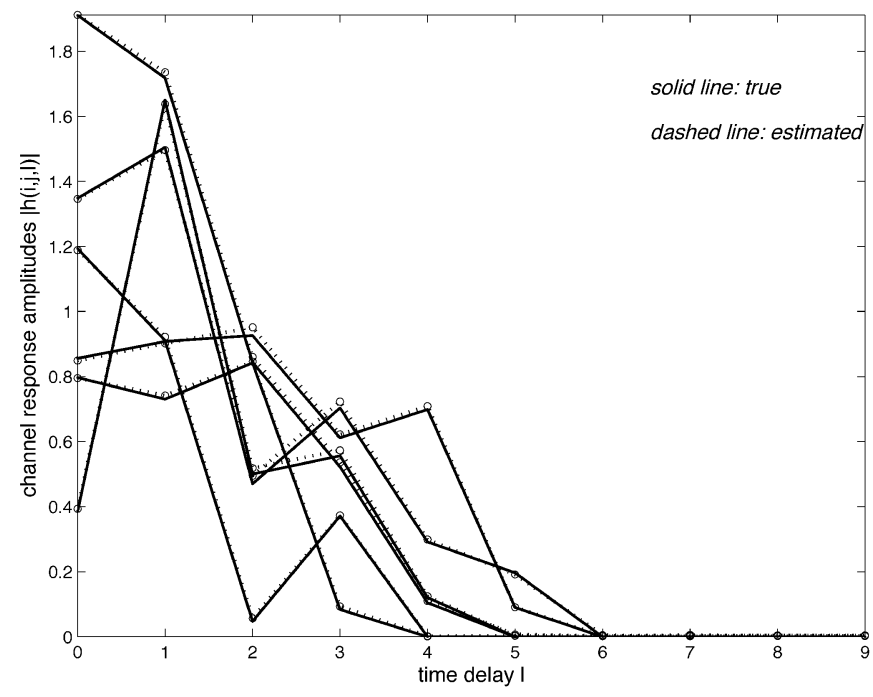

Fig. 5. Channel response amplitudes $(\mathrm{SNR}=28.5 \mathrm{~dB})$.

by Monte Carlo test on 80000 and 20000 symbol blocks for BPSK and 16-QAM, respectively. Since the test is based 


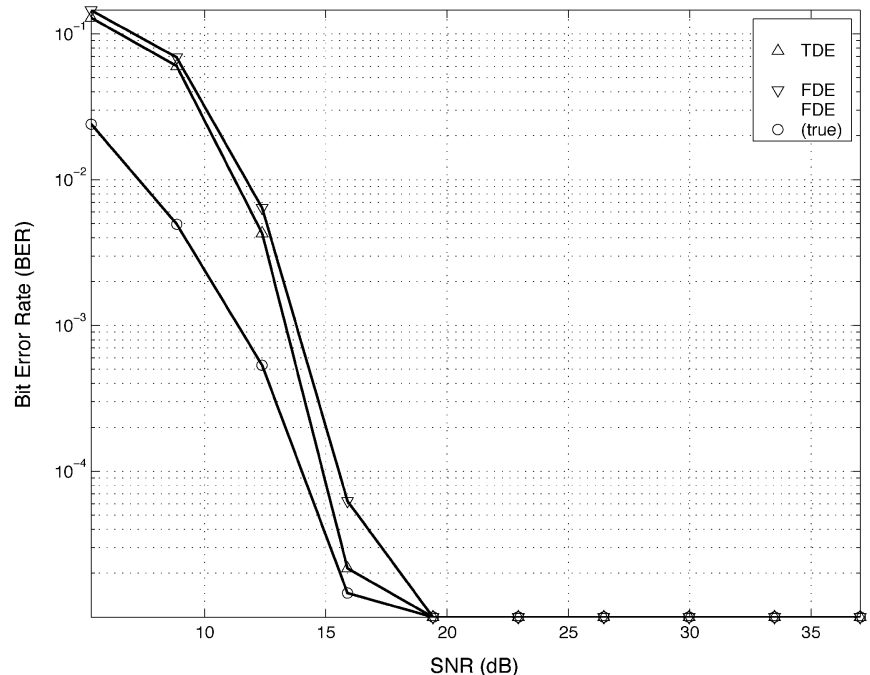

Fig. 6. BER versus SNR (BPSK).

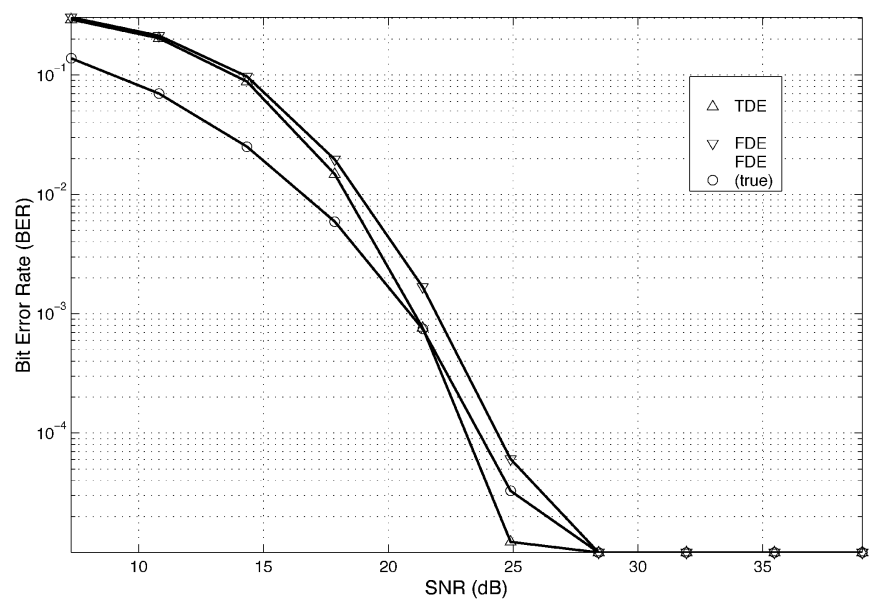

Fig. 7. BER versus SNR (16-QAM).

on finite number of samples, a very small $\left(<10^{-5}\right)$ BER is meaningless, and therefore, it is set to be $10^{-5}$.

Example 4-Multipath Channel Estimation: In a two-user, three-antennas system, the multipath channel length are assumed to be not longer than 4 , and the channel coefficients are generated randomly. Assume that the transmitting filter is a raised cosine function limited in $5 T$, where $T$ is the symbol period. The discrete version of it is $(1.0000,0.9487,0.8057$, $0.6002,0.3721,0)$. The composite channel lengths are then not longer than 9. The matrix $\mathbf{A}$ is

$$
\mathbf{A}=\left[\begin{array}{cccc}
1.0000 & 0 & 0 & 0 \\
0.9487 & 1.0000 & 0 & 0 \\
0.8057 & 0.9487 & 1.0000 & 0 \\
0.6002 & 0.8057 & 0.9487 & 1.0000 \\
0.3721 & 0.6002 & 0.8057 & 0.9487 \\
0 & 0.3721 & 0.6002 & 0.8057 \\
0 & 0 & 0.3721 & 0.6002 \\
0 & 0 & 0 & 0.3721 \\
0 & 0 & 0 & 0
\end{array}\right] .
$$

The OFDM block length is $N=32$, and the input signal is BPSK. To compute the statistical auto-correlation matrix, 150

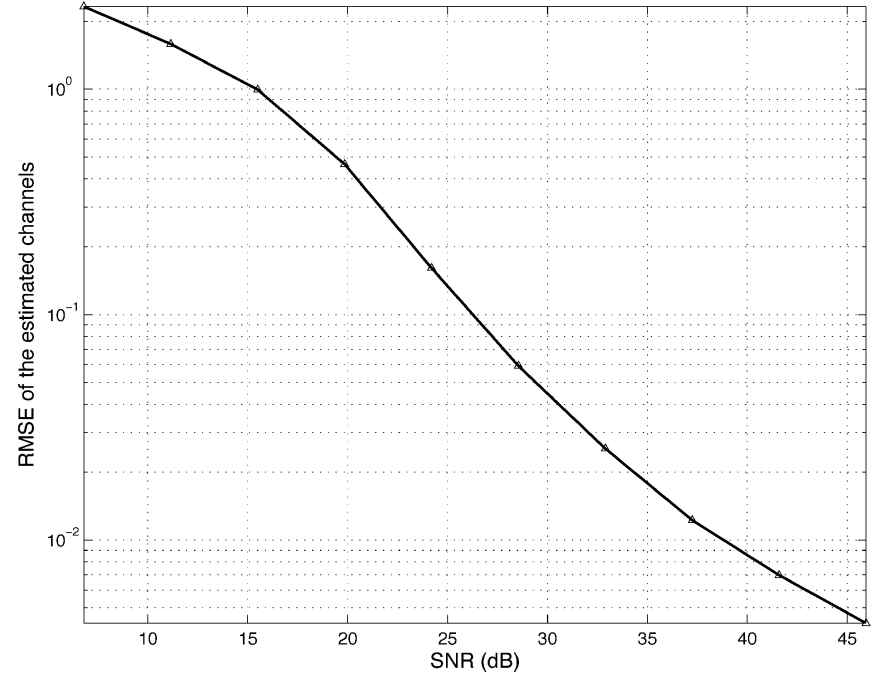

Fig. 8. RMSE of the estimated multipath channel responses.

blocks are used. Fig. 8 shows the RMSE of the estimated multipath channels versus the SNR. The result is averaged over 50 Monte Carlo realizations. This example verifies the correctness of the SS method.

\section{B. Discussions}

It is proved by theory and supported by simulation that the proposed method overcomes two major drawbacks of known SS methods for a general MIMO system, i.e., sensitive to order overestimation and polynomial matrix unidentifiability. The advantages for the SS method, such as simple structure and good performance, are kept. Sensitivity to order overestimation is also a major problem for almost all known SOS-based blind methods, such as the linear prediction (LP) approaches [4], [20], [22], [29], [31] and the outer product decomposition (OPD) [22], [23]. The LP and OPD algorithms are based on a noise-free assumption, and therefore, their performances are very sensitive to observation noise. It has been pointed out in [24], [25], and [36] that LP's claimed robustness to channel order overestimation does not hold when SOS contains estimation errors. It was shown in [20] that the LP algorithm can achieve acceptable performance when the assumed order equals that provided by an order detection criterion overestimated by a few (one or two) taps only. This means that the LP algorithm is not fully robust to order overestimation.

If the system uses CP-OFDM other than ZP-OFDM, it is more difficult to estimate the channels since there is IBI. An SS method for a single-user single-antenna CP-OFDM system is proposed in [13]. It seems difficult to extend the method for the MUMA-OFDM system.

\section{CONCLUSIONS}

In this paper, a subspace-based blind method has been proposed to estimate the channel responses of a multiuser and multiantenna OFDM uplink system. It gives estimations to all channel responses subject to a scalar matrix ambiguity. Unlike the subspace method for general MIMO systems, the proposed method does not need precise channel order information 
and only requires an upper bound for the orders, which can be obtained from some a priori knowledge of propagation conditions in wireless communications. Furthermore, a method is proposed to resolve the scalar ambiguity matrix by using few pilot symbols, provided that the number of users is smaller than that of pilot symbols. Equalization methods are discussed based on the estimated channels. By using partial knowledge of the channels, an eight subspace method is also proposed to reduce the computational complexity. Simulations have shown that the methods are effective and robust.

\section{ACKNOWLEDGMENT}

The authors would like to thank the associate editor and the anonymous reviewers for their invaluable comments.

\section{REFERENCES}

[1] S. M. Alamouti, "A simple transmit diversity technique for wireless communications," IEEE J. Select. Areas Commun., vol. 16, pp. 1451-1458, Oct. 1998.

[2] H. Bolcskei, R. W. Heath Jr., and A. J. Paulraj, "Blind channel identification and equalization in OFDM-based multi-antenna systems," IEEE Trans. Signal Processing, vol. 50, pp. 96-109, Jan. 2002.

[3] J. Chuang and N. Sollenberger, "Beyond 3G: wideband wireless data access based on OFDM and dynamic packet assignment," IEEE Commun. Mag., vol. 32, no. 1, pp. 78-87, 2000.

[4] G. B. Giannakis, Y. Hua, P. Stoica, and L. Tong, Signal Processing Advances in Wireless and Mobile Communications. London, U.K.: Prentice-Hall, 2001, vol. 1, Trends in Channel Estimation and Equalization.

[5] J. Heiskala and J. Terry, OFDM Wireless LANs: A Theoretical and Practical Guide. Indianapolis, IN: Sams, 2002.

[6] Y. Li, J. C. Chung, and N. R. Sollenberger, "Transmitter diversity for OFDM systems and its impact on high-rate data wireless networks," IEEE J. Select. Areas Commun., vol. 17, pp. 1233-1243, Sept. 1999.

[7] Y. Li, N. Seshadri, and S. Ariyavisitakul, "Channel estimation for OFDM systems with transmitter diversity in mobile wireless channels," IEEE J. Select. Areas Commun., vol. 17, pp. 461-471, Apr. 1999.

[8] Z. Liu and G. B. Giannakis, "Space-time block coded multiple access through frequency-selective fading channels," IEEE Trans. Commun., vol. 49, pp. 1033-1044, June 2001.

[9] Z. Liu, G. B. Giannakis, S. Barbarossa, and A. Scaglione, "Transmit antennae space-time block coding for generalized OFDM in the presence of unknown multipath," IEEE J. Select. Areas Commun., vol. 19, pp. $1352-1364$, Sept. 2001

[10] H. Sampath, S. Talwar, J. Tellado, V. Erceg, and A. Paulraj, "A fourthgeneration MIMO-OFDM braodband wireless system: design, performance, and field trial results," IEEE Commun. Mag., pp. 143-149, Sept. 2002.

[11] A. Sklavos, T. Weber, E. Costa, H. Haas, and E. Schulz, "Joint detection in multi-antenna and multi-user OFDM systems," in Multi-Carrier Spread-Spectrum and Related Topics, K. Fazel and S. Kaiser, Eds. Boston, MA: Kluwer, 2002.

[12] S. Zhou, B. Muquet, and G. B. Giannakis, "Subspace-based (semi-) blind channel estimation for block precoded space-time OFDM," IEEE Trans. Signal Processing, vol. 50, pp. 1215-1228, May 2002.

[13] B. Muquet, M. D. Courville, and P. Duhamel, "Subspace-based blind and semi-blind channel estimation for OFDM systems," IEEE Trans. Signal Processing, vol. 50, pp. 1699-1712, July 2002.

[14] B. Muquet, Z. Wang, G. B. Giannakis, M. D. Courville, and P. Duhamel, "Cyclic prefixing or zero padding for wireless multicarrier transmissions," IEEE Trans. Communications, vol. 50, no. 12, pp. 2136-2148, 2002.

[15] A. Scaglione, G. B. Giannakis, and S. Barbarossa, "Redundant filterbank precoders and equalizers-part II: blind channel estimation, synchronization and direct equalization," IEEE Trans. Signal Processing, vol. 47, no. 7, pp. 2002-2007, 1999.

[16] H. Sari, G. Karam, and I. Jeanclaude, "Transmission techniques for digital terrestrial TV broadcasting," IEEE Commun. Mag., pp. 100-109, Feb. 1995.

[17] B. Gyselinckx and M. Engels, "Training sequence versus cyclic prefix-A new look on single carrier communication," IEEE Commun. Lett., vol. 5, pp. 292-294, July 2001.
[18] T. Pollet, M. V. Bladel, and M. Moeneclaey, "BER sensitivity of OFDM systems to carrier frequency offset and wiener phase noise," IEEE Trans. Commun., vol. 43, pp. 191-193, Feb. 1995.

[19] K. Abed-Meraim, P. Loubaton, and E. Moulines, "A subspace algorithm for certain blind identification problem," IEEE Trans. Inform. Theory, vol. 32, pp. 499-511, Apr. 1997.

[20] - "Prediction error method for second-order blind identification," IEEE Trans. Signal Processing, vol. 45, pp. 694-705, Mar. 1997.

[21] A. Cichocki and S. I. Amari, Adaptive Blind Signal and Image Processing. New York: Wiley, 2002.

[22] Z. Ding and Y. Li, Blind Equalization and Identification. New York: Marcel Dekker, 2001.

[23] Z. Ding, "Matrix outer product decomposition method for blind multiple channel identification," IEEE Trans. Signal Processing, vol. 45, pp. 3053-3061, Dec. 1997.

[24] H. Gazzah, P. A. Regalia, J. P. Delmas, and K. Abed-Meraim, "A blind multichannel identification algorithm robust to order overestimation," IEEE Trans. Signal Processing, vol. 50, pp. 1449-1458, June 2002.

[25] A. Gorokhov and P. Loubaton, "Blind identification of MIMO-FIR systems: a generalized prediction approach," Signal Process., vol. 73, pp. $105-124,1999$.

[26] Y. A. Huang and J. Benesty, "Adaptive multi-channel least mean square and Newton algorithms for blind channel identification," Signal Process., vol. 82, pp. 1127-1138, 2002.

[27] H. Liu, G. Xu, L. Tong, and T. Kailath, "Recent development in blind channel equalization: from cyclostationarity to subspaces," Signal Process., vol. 50, pp. 83-99, 1996.

[28] E. Moulines, P. Duhamel, J. F. Cardoso, and S. Mayrargue, "Subspace methods for the blind identification of multichannel FIR filters," IEEE Trans. Signal Processing, vol. 43, pp. 516-525, Feb. 1995.

[29] D. Slock, "Blind fractionally-spaced equalization, perfect reconstruction filterbanks, and multilinear prediction," in Proc. IEEE ICASSP Conf., Adalaide, Australia, 1994.

[30] L. Tong, G. Xu, and T. Kailath, "Blind identification and equalization based on second-order statistics: a time domain approach," IEEE Trans. Inform. Theory, vol. 40, pp. 340-349, Mar. 1994.

[31] J. K. Tugnait, "On linear predictors for MIMO channels and related blind identification and equalization," IEEE Signal Processing Lett., vol. 5, pp. 289-291, Nov. 1998.

[32] J. K. Tugnait and B. Huang, "Blind estimation and equalization of MIMO channels via multidelay whitening," IEEE J. Select. Areas Commun., vol. 19, pp. 1507-1519, Oct. 2001.

[33] W. Qiu and Y. Hua, "Performance analysis of the subspace method for blind channel identification," Signal Process., vol. 50, pp. 71-81, 1996.

[34] S. Roy and C. Y. Li, "A subspace blind channel estimation method for OFDM systems without cyclic prefix," IEEE Trans. Wireless Commun., vol. 1 , pp. $572-579$, July 2002 .

[35] M. Wax and T. Kailath, "Detection of signals by information theoretic criteria," IEEE Trans. Acoust., Speech, Signal Processing, vol. ASSP-32, pp. 387-392, Apr. 1985.

[36] A. P. Liavas, P. A. Regalia, and J. P. Delmas, "On the robustness of the linear prediction method for blind channel identification with respect to effective channel undermodeling/overmodeling," IEEE Trans. Signal Processing, vol. 48, pp. 1477-1481, May 2000.

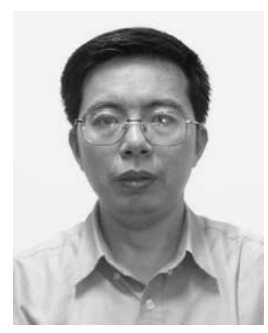

Yonghong Zeng (M'99) received the B.S. degree in mathematics from the Peking University, Beijing, China, in 1983 and the M.S. degree in applied mathematics and $\mathrm{Ph} . \mathrm{D}$. degree in computer science and technology from the National University of Defense Technology, Changsha, China, in 1986 and 1998, respectively.

Since 1986, he has been with the Department of System Engineering and Mathematics, National University of Defense Technology, where he has served as an associate professor since 1993. He joined the School of Electrical and Electronic Engineering, Nanyang Technological University, Singapore, to work as a research fellow in 1999. Currently, he is working for the Department of Electrical and Electronic Engineering, the University of Hong Kong. His main research interests include signal processing for communication, radar, and array signal processing, algorithms and transforms for signal processing, and parallel computing. He has published five books and a number of papers.

Dr. Zeng received the ministry-level Scientific and Technological Development Awards in China four times. 


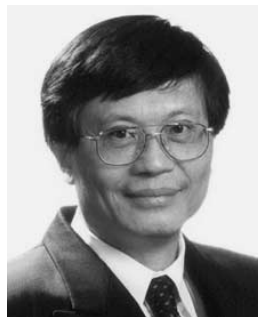

Tung-Sang Ng (S'74-M'78-SM'90-F'03) received the B.Sc.(Eng.) degree from the University of Hong Kong in 1972 and the M.Eng.Sc. and Ph.D. degrees from the University of Newcastle, Callaghan, Australia, in 1974 and 1977, respectively, all in electrical engineering.

He worked for BHP Steel International,Newcastle, Australia, and The University of Wollongong, Wollongong, Australia, after graduation for 14 years and returned to Hong Kong in 1991, taking up the position of Professor and Chair of Electronic Engineering. He has been Head of Department of Electrical and Electronic Engineering since 2000. His current research interests include wireless communication systems, spread spectrum techniques, CDMA, and digital signal processing. He has published over 250 international journal and conference papers.

Dr. Ng was the General Chair of ISCAS'97 and the VP-Region 10 of IEEE CAS Society in 1999 and 2000. He was an Executive Committee Member and a Board Member of the IEE Informatics Divisional Board from 1999 to 2001 and was an ordinary member of IEE Council from 1999 to 2001. He received the Honorary Doctor of Engineering Degree from the University of Newcastle, in August 1997 for his services to higher education generally and to engineering education specifically. He received the Senior Croucher Foundation Fellowship in 1999 and the IEEE Third Millenium medal in February 2000. He is a Fellow of HKIE. 